\title{
Neoplasia de próstata en trabajadores expuestos al cadmio y/o sus compuestos: revisión sistemática
}

\author{
Prostatic Neoplasm in Workers Exposed to Cadmium and/or its Compounds: \\ Systematic Review
}

\author{
Ángela Kenia Baines Camps', Alberto Daniel Levy Moshe², María Fernanda Visconti Pimentel ${ }^{2}$, Javier Sanz- \\ Valero $^{3}$ \\ 1. Unidad Docente de Medicina del Trabajo Comunidad Autónoma del País Vasco, Vitoria, España. \\ 2. Unidad Docente de Medicina del Trabajo de Madrid, Madrid, España. \\ 3. Universidad Miguel Hernández, Departamento de Salud Pública e Historia de la Ciencia, Alicante, España.
}

Recibido: 07-03-2018

Aceptado: 10-04-2019

\section{Correspondencia}

María Fernanda Visconti Pimentel

Correo electrónico: maria.visconti@gmail.com

Este trabajo se ha desarrollado dentro del Programa Científico de la Escuela Nacional de Medicina del Trabajo del Instituto de Salud Carlos III en convenio con la Unidad Docente de Medicina del Trabajo del País Vasco y la Unidad Docente de Medicina del Trabajo de la Comunidad de Madrid. Madrid. España.

Resumen

Introducción: La neoplasia de próstata es la enfermedad maligna más común de los países desarrollados y la segunda causa de muerte a nivel mundial.

Objetivos: Revisar la literatura científica existente sobre la aparición de neoplasias de próstata asociada a la exposición laboral al cadmio y/o sus compuestos.

Métodos: Revisión sistemática de la literatura científica recogida en las bases de datos bibliográficas MEDLINE (vía PubMed), The Cochrane Library, Scopus y Web of Science. Los términos utilizados, como descriptores y texto libre en título y resumen, fueron: "Cadmium", "Cadmium Compounds", "Cadmium Radioisotopes", "Cadmium Isotopes», "Cadmium Chloride», "Cadmium Poisoning», "Itai Itai", "Prostatic Neoplasms", "Prostatic Neoplasm», "Prostate Neoplasm», "Prostate Cancer», "Cancer of the Prostate», "Prostatic Cancer", "Cancer of Prostate» $y$ "Occupational Exposure». Se utilizaron los filtros (límites) "humanos" y "adultos" (19+ años).

Resultados: Se recuperaron 114 referencias, de las que se pudieron obtener a texto completo 14 artículos tras aplicar los criterios de inclusión y exclusión. En ellos se describe la asociación entre exposición laboral al cadmio y el desarrollo de neoplasia de próstata.

Conclusiones: A pesar de obtener unos resultados que evalúan el desarrollo de neoplasia de próstata tras la exposición al cadmio o a sus compuestos, no existe suficiente evidencia que demuestre su efecto

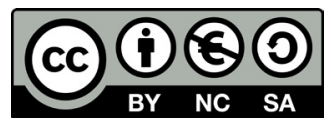

https://creativecommons.org/licenses/by-nc-sa/4.0/ 
carcinogénico en el ámbito laboral, por lo que serían necesarios nuevos estudios que aporten evidencia en relación a este tema.

Med Segur Trab (Internet). 2019;65(254):59-72

Palabras clave: Cadmio; compuestos del cadmio; exposición laboral; neoplasia de próstata.

\section{Abstract

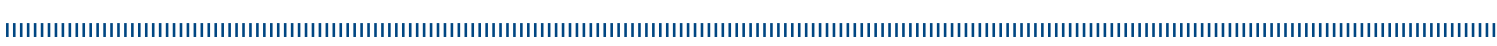

Introduction: Prostate neoplasm is the most common malignant disease in developing countries and the second cause of death worldwide.

objectives: To review the existing scientific literature on the appearance of prostate neoplasms associated with occupational exposure to cadmium and/or its compounds.

Metbods: Systematic review of the scientific literature collected in the MEDLINE bibliographic databases (via PubMed), The Cochrane Library, Scopus and Web of Science. The terms used, as descriptors and free text in title and summary, were: "Cadmium", "Cadmium Compounds", "Cadmium Radioisotopes", "Cadmium Isotopes», "Cadmium Chloride», "Cadmium Poisoning», «Itai Itai», "Prostatic Neoplasms», "Prostatic Neoplasm», "Prostate Neoplasm», "Prostate Cancer", "Cancer of the Prostate», "Prostatic Cancer», "Cancer of Prostate» and «Occupational Exposure». The filters (limits) «humans» and «adults» (19+ years) were considered.

Results: 114 references were retrieved, 14 articles of them in full text after applying the inclusion and exclusion criteria, in which the association between occupational exposure to cadmium and the development of prostate neoplasm is explained.

Conclusions: Despite the results obtained that evaluate the development of prostate cancer after exposure to cadmium or its compounds, not enough evidence to demonstrate its carcinogenic effect in the workplace has been found, so new studies that provide evidence in relation to this topic should be carried out.

Med Segur Trab (Internet). 2019;65(254):59-72

Keywords: Cadmium; cadmium compounds; occupational exposure; prostate neoplasia. 


\section{INTRODUCCIÓN}

La neoplasia de próstata es la enfermedad maligna más común de los países desarrollados y la sexta causa de muerte tras el cáncer de pulmón a nivel mundial ${ }^{1}$. La tasa de mortalidad para la neoplasia de próstata varía según el grupo étnico, detectándose altas tasas en afroamericanos y caucásicos en comparación con los asiáticos. Estas diferencias son debidas a susceptibilidad genética, exposición a factores de riesgo externos, diferencias en el sistema de salud, el registro de la neoplasia como tal en la historia clínica o una combinación de estos factores ${ }^{2}$. En el año 2015 más de 220.800 hombres fueron diagnosticados de neoplasia de próstata y 27.540 murieron según las estadísticas de los Estados Unidos de Norteamérica (EE.UU.) ${ }^{3}$. En ese mismo año fueron diagnosticados más de 33.300 varones de neoplasia de próstata en España, un 22.4\% sobre el total de neoplasias detectadas ${ }^{4}$. La etiología de la neoplasia de próstata comprende múltiples factores, dentro de los cuales pueden ser implicados la obesidad, los andrógenos, la dieta rica en grasas y el cadmio. La exposición al licopeno y a la vitamina $\mathrm{E}$ han demostrado ser protectoras frente a este tipo de cáncer ${ }^{5,6}$.

El cadmio es un elemento metálico que se encuentra en la naturaleza en pequeñas concentraciones, representando alrededor de $0.1-0.5 \mathrm{mg} / \mathrm{kg}$ de la corteza terrestre, y se presenta principalmente en asociación con minerales de zinc (en una proporción del $0.33 \%$ ), pero también con minerales de plomo o cobre ${ }^{7}$. El contacto con este metal puede ocurrir a través de fuentes que incluyen el tabaquismo, la dieta (mediante vegetales que adquieren el cadmio de los suelos), la contaminación ambiental y la exposición en el medio laboral al inhalar polvo o humos que lo contengan ${ }^{8,9}$. El cadmio tiene una vida media en el organismo de entre 10 y 30 años y puede presentar una gran variedad de efectos negativos en la salud de los humanos. Además de ser carcinogénico, se ha relacionado con la aparición de osteoporosis y fracturas óseas, diabetes mellitus tipo 2, enfermedades renales y cardiovasculares?

Sus primeros usos, a principios del siglo $\mathrm{XX}$, fueron como pigmento para pinturas, en amalgamas dentales (en cantidades más reducidas) y como sustituto del estaño durante la Segunda Guerra Mundial. Desde entonces, el cadmio ha sido utilizado en su práctica totalidad para la fabricación de baterías y pigmentos, en la galvanoplastia y los revestimientos, y como estabilizador de plásticos y aleaciones. Casi todos estos usos han disminuido hacia el final del siglo $\mathrm{XX}$, siendo actualmente su principal utilización la fabricación de baterías, que pasó de un $8 \%$ en 1970 a un $75 \%$ en el año $2000^{10}$. En 2003 , la Unión Europea (UE) adoptó la resolución sobre la Restricción del Manejo de Sustancias Peligrosas, por la aprobación de la cual los Estados miembros garantizan que, a partir de julio de 2006, los nuevos aparatos eléctricos y electrónicos que se pongan en el mercado no contendrán cadmio, así como tampoco plomo, mercurio, cromo hexavalente, polibromobifenilos (PBB) o polibromodifeniléteres (PBDE), salvo para algunas aplicaciones que quedan exceptuadas de lo dispuesto por no existir alternativas adecuadas y más seguras ${ }^{11}$.

Se han descrito varios mecanismos responsables de la carcinogenicidad de la exposición al cadmio, que incluyen la inducción del estrés oxidativo, la supresión de la reparación del ADN, alteraciones en la metilación del ADN, inhibición de la apoptosis, activación de protooncogenes, inactivación de genes supresores de tumores y disrupción de la adhesión celular. Estudios experimentales evidencian que un exceso de exposición a estrógenos puede provocar neoplasia de próstata. Se ha demostrado que el cadmio puede presentar propiedades estrogénicas y desencadenar procesos mediados por receptores $\alpha$ en el tejido prostático, activando la proliferación celular ${ }^{12}$.

Varios estudios epidemiológicos ${ }^{13-21}$ investigan la asociación entre la exposición ocupacional al cadmio y el desarrollo de neoplasia de próstata, sin embargo se han presentado hallazgos inconsistentes. Algunos estudios han demostrado una relación significativa ${ }^{13-16}$, otros una asociación débil ${ }^{17,18}$, mientras que otros, por el contrario, no demostraron ninguna asociación ${ }^{19,20}$. La International Agency for Research on Cancer 
(IARC) reporta en su informe de 2018 resultados contradictorios sobre la asociación entre la exposición al cadmio en el medio laboral y el desarrollo de neoplasias de próstata. Los incrementos que se observaron en unos primeros estudios de cohortes no pudieron confirmarse en estudios posteriores. Sin embargo, un estudio de casos y controles sugiere que esta asociación existe ${ }^{21}$.

Por ello, se consideró de interés realizar una revisión con el objetivo de examinar la literatura científica sobre la exposición laboral al cadmio y/o sus compuestos en relación con la aparición de neoplasia de próstata.

\section{MATERIAL Y MÉTODOS}

\section{Diseño}

Estudio descriptivo transversal y análisis crítico de los trabajos recuperados mediante revisión sistemática.

\section{Fuente de obtención de los datos}

Los datos se obtuvieron mediante consulta directa, vía Internet, a la literatura científica contenida en las bases de datos MEDLINE (vía PubMed), The Cochrane Library, Scopus y Web of Science.

\section{Tratamiento de la información}

Para definir los términos de la búsqueda se consultó el Thesaurus desarrollado por la U.S. National Library of Medicine (MeSH), considerándose adecuados "Cadmium", "Cadmium Compounds", "Cadmium Radioisotopes", "Cadmium Isotopes", "Cadmium Chloride», "Cadmium Poisoning», "Itai Itai», "Prostatic Neoplasms», "Prostatic Neoplasm», "Prostate Neoplasm», "Prostate Cancer», "Cancer of the Prostate», "Prostatic Cancer», "Cancer of Prostate» y "Occupational Exposure», como descriptores y texto libre en título y resumen. La ecuación de búsqueda principal se desarrolló, con la utilización de conectores booleanos, para su empleo en la base de datos MEDLINE, vía PubMed. Se utilizaron los filtros (límites) «humanos» y "adultos 19+ años». Esta misma estrategia se adaptó a las características del resto de bases de datos consultadas; ver tabla I.

La búsqueda se realizó desde la primera fecha disponible, de acuerdo con las características de cada base de datos, hasta noviembre de 2018 y se completó con el examen del listado bibliográfico de los artículos que fueron seleccionados.

\section{Selección de estudios}

Se eligieron para su revisión los artículos que cumplieron los siguientes criterios de inclusión: artículos originales publicados en revistas revisadas por pares que fueran estudios observacionales y que existiera efecto causal entre la exposición al cadmio y/o sus compuestos y la neoplasia de próstata por exposición laboral en personas adultas. Al aplicar los criterios de exclusión fueron eliminados aquellos artículos con idiomas diferentes a los establecidos (inglés, portugués y castellano) y no encontrados a texto completo.

La selección de los artículos pertinentes se realizó por los autores de la presente revisión. Para dar por válida la inclusión de los artículos se estableció que la valoración de la concordancia (índice Kappa) entre tres de los autores (ADLM/MFVP/AKBC) debía ser superior a 0,60 (buena o muy buena fuerza de concordancia) ${ }^{22}$. Siempre que se cumpliera esta condición, las posibles discordancias se solucionarían mediante la consulta del cuarto autor (JSV) y posterior consenso entre los autores. 
Tabla I. Estrategia de búsqueda adaptada a cada una de las bases de datos bibliográficas consultadas.

\begin{tabular}{|c|c|}
\hline Bases de datos & Ecuaciones de búsqueda \\
\hline $\begin{array}{l}\text { MEDLINE } \\
\text { (Pubmed) }\end{array}$ & 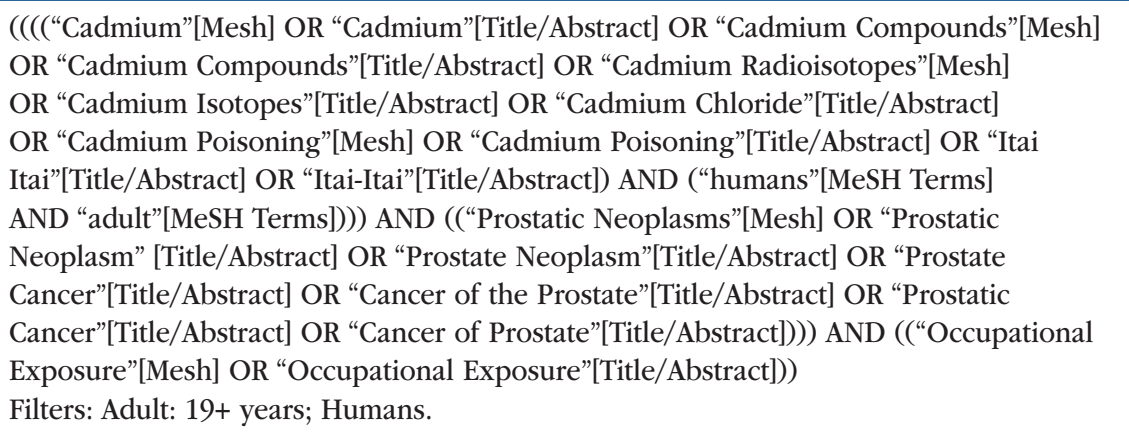 \\
\hline $\begin{array}{l}\text { The Cochrane } \\
\text { Library }\end{array}$ & $\begin{array}{l}\text { (MeSH descriptor: [Cadmium] OR [Cadmium Compounds] OR ("Cadmium" OR } \\
\text { "Cadmium Compounds" OR "Cadmium Compounds" OR "Cadmium Radioisotopes" } \\
\text { OR "Cadmium Radioisotopes" OR "Cadmium Isotopes" OR "Cadmium Chloride" OR } \\
\text { "Cadmium Poisoning" OR "Cadmium Poisoning" OR "Itai Itai" OR "Itai-Itai"):ti,ab,kw) } \\
\text { AND (MeSH descriptor: [Prostatic Neoplasms] OR ("Prostatic Neoplasms" OR "Prostatic } \\
\text { Neoplasm" OR "Prostate Neoplasm" OR "Prostate Cancer" OR "Cancer of the Prostate" } \\
\text { OR "Prostatic Cancer" OR "Cancer of Prostate"):ti,ab,kw) AND (MeSH descriptor: } \\
\text { [Occupational Exposure] OR ((“Occupational Exposure"):ti,ab,kw) }\end{array}$ \\
\hline Scopus & $\begin{array}{l}\text { ( TITLE-ABS-KEY ( "Cadmium" OR "Cadmium Compounds" OR "Cadmium Compounds" } \\
\text { OR "Cadmium Radioisotopes" OR "Cadmium Radioisotopes" OR "Cadmium Isotopes" } \\
\text { OR "Cadmium Chloride" OR "Cadmium Poisoning" OR "Cadmium Poisoning" OR } \\
\text { "Itai Itai" OR "Itai-Itai" ) AND TITLE-ABS-KEY ("Prostatic Neoplasms" OR "Prostatic } \\
\text { Neoplasm" OR "Prostate Neoplasm" OR "Prostate Cancer" OR "Cancer of the Prostate" } \\
\text { OR "Prostatic Cancer" OR "Cancer of Prostate" ) ) AND ( LIMIT-TO ( DOCTYPE , "ar") ) }\end{array}$ \\
\hline Web of Science & $\begin{array}{l}\text { TEMA: ("Cadmium" OR "Cadmium Compounds" OR "Cadmium Compounds" OR } \\
\text { "Cadmium Radioisotopes" OR "Cadmium Radioisotopes" OR "Cadmium Isotopes" OR } \\
\text { "Cadmium Chloride" OR "Cadmium Poisoning" OR "Cadmium Poisoning" OR "Itai Itai" } \\
\text { OR "Itai-Itai") AND TEMA: ("Prostatic Neoplasms" OR "Prostatic Neoplasm" OR "Prostate } \\
\text { Neoplasm" OR "Prostate Cancer" OR "Cancer of the Prostate" OR "Prostatic Cancer" OR } \\
\text { "Cancer of Prostate") }\end{array}$ \\
\hline
\end{tabular}

\section{Evaluación de la calidad metodológica}

La calidad de los artículos seleccionados se valoró utilizando como apoyo las pautas para informar los estudios observacionales STROBE (STrengthening the Reporting of OBservational studies in Epidemiology $)^{23}$, que contiene una lista de 22 puntos de control esenciales que deben describirse durante la publicación de estos documentos. Para cada artículo seleccionado se asignó un punto por cada ítem presente (en caso de no ser aplicable no puntuaba). Cuando un ítem estaba compuesto por varios puntos, éstos se evaluaron de forma independiente, dándole el mismo valor a cada uno de ellos y posteriormente se realizó un promedio (siendo éste el resultado final de ese ítem), de tal forma que en ningún caso se pudiera superar la puntuación de un punto por ítem.

\section{Extracción de los datos}

El control de la información extraída de los estudios revisados se realizó mediante dobles tablas que permitían la detección de los errores y la corrección mediante nueva consulta de los originales.

Para determinar la actualidad de los artículos se calculó el semiperiodo de BurtonKleber (mediana de la edad) y el índice de Price (porcentaje de artículos con edad inferior a los 5 años).

Los estudios se agruparon según las variables a estudio, con el fin de sistematizar y facilitar la comprensión de los resultados, considerando los siguientes datos: primer autor 
de la referencia bibliográfica y año de publicación, tipo de estudio y población, período y localización donde se realizó, agente(s) de exposición, ocupación, tiempo de exposición, efecto estudiado y resultados principales.

\section{RESULTADOS}

Después de aplicar los criterios de búsqueda descritos se recuperaron un total de 114 referencias. De éstas, tras eliminar aquellas que estaban duplicadas y aplicar los criterios de inclusión y exclusión (ver figura 1), se lograron recuperar catorce artículos ${ }^{24-37}$ a texto completo (ver tablas III, IV, V), procedentes de MEDLINE ( $n=6 ; 42.85 \%)$, Scopus $(n=6 ; 42.85 \%)$ y Web of Science $(n=2 ; 14.3 \%)$.

Figura 1. Identificación y selección de estudios.

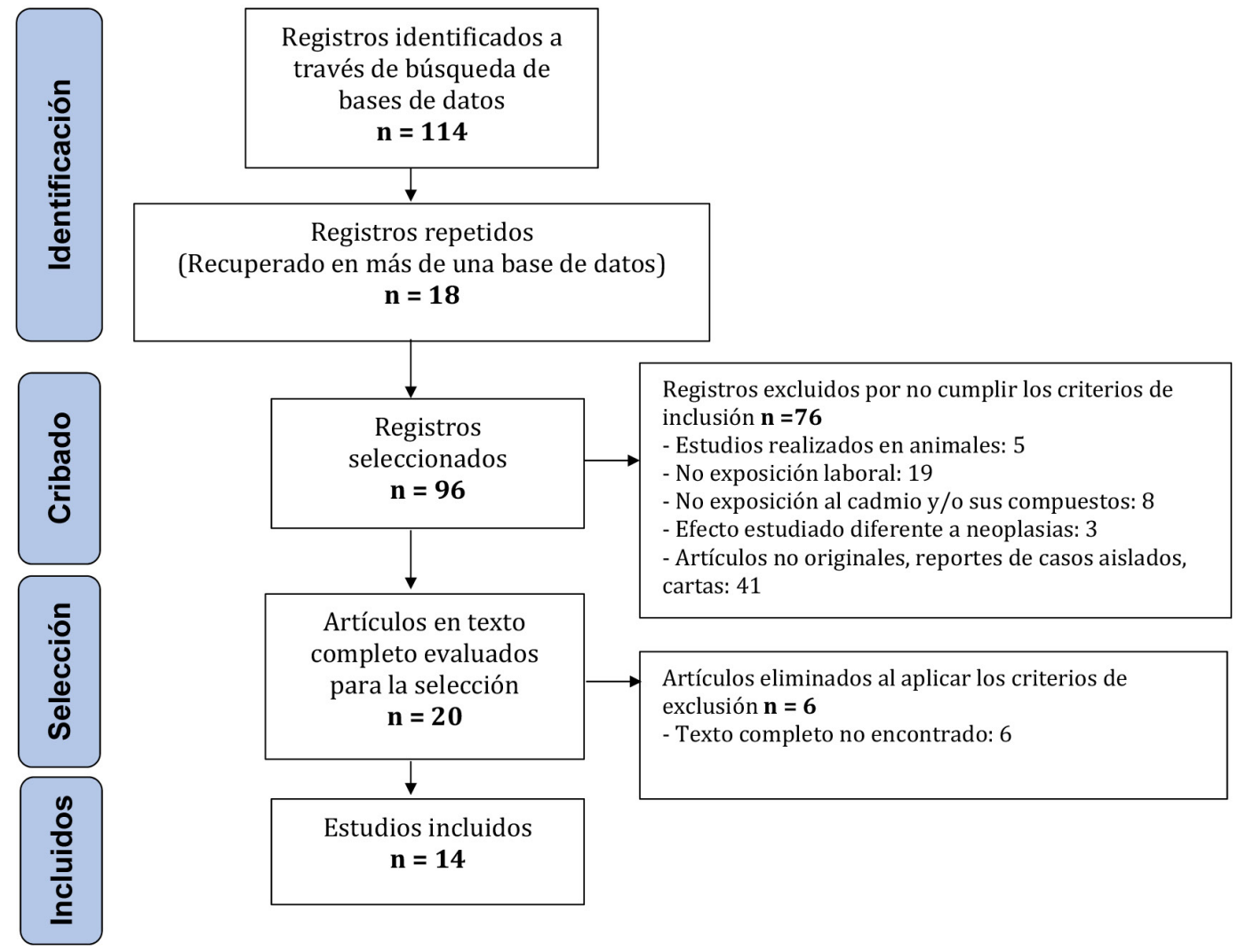

Al calcular la concordancia entre los evaluadores sobre la pertinencia de los estudios seleccionados se obtuvieron unos valores que oscilaron entre $0,70(\mathrm{p}<0,008)$ y 0,85 ( $\mathrm{p}$ $=0,002$ ). Los artículos elegidos presentaron una obsolescencia, según el índice de BurtonKleber, igual a 25 años, con un índice de Price del 14,3\%.

Después de la evaluación de la calidad de los artículos seleccionados por el cuestionario STROBE, las puntuaciones oscilaron entre 8,16 y 19,26 (ver tabla II).

Los trabajos revisados incluyeron $\operatorname{cinco}^{26,34,35,36,37}$ estudios de cohortes $(35,71 \%)$, tres $^{26,35,37}$ de los cuales eran retrospectivos, lo cual dificultó establecer la relación causal entre exposición y efecto; nueve $24,25,27,28,29,30,31,32,33$ fueron casos y controles $(64,28 \%)$.

El estudio de Checkoway et al. ${ }^{31}$, es el que presentó menor tamaño muestral $(\mathrm{n}=104)$, mientras que el de mayor tamaño muestral fue el publicado por Sharma-Wagner et al. ${ }^{26}$ $(n=36.269)$. La población estudiada en trece de los catorce trabajos $(92,85 \%)$ estuvo formada por hombres exclusivamente ${ }^{24-36}$ y en uno de los estudios $(7,15 \%)$ fueron incluidos individuos de ambos sexos ${ }^{37}$. 
Tabla II. Evaluación de la calidad metodológica de los estudios a través de los 22 ítems de valoración de la guía STROBE.

\begin{tabular}{|c|c|c|c|c|c|c|c|c|c|c|c|c|c|c|c|c|c|c|c|c|c|c|c|c|}
\hline \multirow{2}{*}{ Referencia } & \multicolumn{24}{|c|}{ Puntuación de los 22 ítems } \\
\hline & 1 & 2 & 3 & 4 & 5 & 6 & 7 & 8 & 9 & 10 & 11 & 12 & 13 & 14 & 15 & 16 & 17 & 18 & 19 & 20 & 21 & 22 & Total & $\%$ \\
\hline $\begin{array}{l}\text { Van Der } \\
\text { Gulden et al. }{ }^{24}\end{array}$ & 1 & 1 & 1 & 1 & 1 & 1 & 1 & 1 & 0 & 0 & 0 & 0.20 & 0.66 & 0.50 & 1 & 1 & 0 & 1 & 1 & 1 & 1 & 0 & 15.30 & 69.54 \\
\hline $\begin{array}{l}\text { Elghany } \\
\text { et al. }{ }^{25}\end{array}$ & 1 & 1 & 1 & 1 & 1 & 1 & 1 & 1 & 1 & 0 & 1 & 0.20 & 0.66 & 0.50 & 1 & 1 & 1 & 1 & 1 & 1 & 0 & 0 & 17.30 & 78.63 \\
\hline $\begin{array}{l}\text { Sharma- } \\
\text { Wagner et al. }\end{array}$ & 1 & 1 & 1 & 0 & 1 & 1 & 0 & 0 & 0 & 0 & 0 & 0.20 & 0 & 0 & 1 & 0.33 & 0 & 1 & 1 & 1 & 0 & 0 & 9.53 & 43.31 \\
\hline $\begin{array}{l}\text { Neslund- } \\
\text { Dudas et } a l .{ }^{27}\end{array}$ & 1 & 1 & 1 & 1 & 1 & 1 & 1 & 1 & 1 & 0 & 1 & 0.60 & 0.33 & 0.50 & 1 & 1 & 0 & 1 & 1 & 1 & 1 & 1 & 18.43 & 83.77 \\
\hline Seidler et al..$^{28}$ & 1 & 1 & 1 & 1 & 1 & 1 & 1 & 1 & 1 & 0 & 1 & 0.50 & 0.33 & 0.50 & 1 & 1 & 0 & 1 & 0 & 1 & 0 & 0 & 15.33 & 69.68 \\
\hline Rooney et al. ${ }^{29}$ & 1 & 1 & 1 & 1 & 1 & 1 & 1 & 1 & 1 & 0 & NA & 0.60 & 0.33 & 0.50 & 1 & 1 & 0 & 1 & 0 & 1 & 0 & 1 & 15.43 & 73.47 \\
\hline Krech et $a l .^{30}$ & 1 & 1 & 0 & 1 & 1 & 1 & 0 & 0 & 0 & 0 & NA & 0.33 & 0.33 & 0 & 1 & 0.50 & 0 & 0 & 0 & 1 & 0 & 0 & 8.16 & 38.85 \\
\hline $\begin{array}{l}\text { Checkoway } \\
\text { et al. } .^{31}\end{array}$ & 1 & 1 & 1 & 1 & 1 & 1 & 1 & 1 & 1 & 0 & 1 & 0 & 0.33 & 0.50 & 1 & 0 & 0 & 1 & 1 & 1 & 0 & 1 & 15.83 & 71.95 \\
\hline $\begin{array}{l}\text { Armstrong } \\
\text { et al. }{ }^{32}\end{array}$ & 1 & 1 & 0 & 1 & 1 & 1 & 0 & 0 & 0 & 0 & 1 & 0.60 & 0.33 & 0 & 1 & 1 & 0 & 1 & 0 & 1 & 0 & 1 & 11.93 & 54.22 \\
\hline Fritschi et $a l^{33}$ & 1 & 1 & 1 & 1 & 1 & 1 & 1 & 1 & 1 & 0 & 0 & 0.75 & 0.66 & 0.50 & 1 & 1 & 0 & 1 & 1 & 1 & 1 & 1 & 17.91 & 81.40 \\
\hline $\begin{array}{l}\text { Sorahan } \\
\text { et al. }{ }^{34}\end{array}$ & 1 & 1 & 1 & 1 & 1 & 1 & 1 & 1 & 0 & 0 & 0 & 0.60 & 0.66 & 1 & 1 & 0.50 & 1 & 1 & 1 & 1 & 1 & 1 & 17.76 & 80.72 \\
\hline $\begin{array}{l}\text { Kazantzis } \\
\text { et al. }{ }^{35}\end{array}$ & 1 & 1 & 1 & 1 & 1 & 1 & 0 & 1 & 0 & 0 & 1 & 0.50 & 0.66 & 0.33 & 1 & 1 & 0 & 1 & 0 & 1 & 0 & 1 & 14.49 & 65.86 \\
\hline $\begin{array}{l}\text { Andersson } \\
\text { et al. }\end{array}$ & 1 & 1 & 1 & 1 & 1 & 1 & 1 & 1 & 1 & 0 & 1 & 0.60 & 0.66 & 1 & 1 & 1 & 1 & 1 & 1 & 1 & 1 & 0 & 19.26 & 87.54 \\
\hline $\begin{array}{l}\text { Sorahan } \\
\text { et al. }\end{array}$ & 1 & 1 & 1 & 1 & 1 & 1 & 1 & & 1 & 0 & 1 & 0.40 & 0.33 & 0.66 & 1 & 0.33 & 1 & 1 & 1 & 1 & 0 & 0 & 15.72 & 71.45 \\
\hline
\end{tabular}

0 = no cumple el ítem ni ninguna de sus partes; 1 = cumple el ítem en su totalidad; 0 a 1 = Cumple parcialmente el ítem; $N A=$ no aplica

Debido a que el efecto analizado en diez de los catorce trabajos fue específicamente la neoplasia de próstata, la población estudiada se compuso únicamente de varones ${ }^{24-33}$. En los otros cuatro estudios se analizó la mortalidad de trabajadores expuestos al cadmio por diversas causas ${ }^{34-37}$, de los cuales el único que incluyó a ambos sexos fue el publicado por Sorahan et al. ${ }^{37}$. Andersson et al..$^{36}$ encontraron una cohorte conformada por trabajadores de ambos sexos, sin embargo las mujeres fueron finalmente excluidas por haber tenido un periodo de exposición muy breve al cadmio.

En cuanto a la procedencia de los estudios seleccionados, cinco fueron llevados a cabo en Reino Unido ${ }^{29,32,34,35,37}$, tres en EE.UU. ${ }^{25,27,31}$, dos en Alemania ${ }^{28,30}$, dos en Suecia ${ }^{26,36}$, uno en Holanda ${ }^{24}$ y uno en Australia ${ }^{33}$.

Sorahan et al. ${ }^{37}$ publicaron el más temprano de los artículos en 1983, mientras que el más reciente de ellos fue publicado por Krech et al. ${ }^{30}$ en 2016 . El trabajo con mayor período de seguimiento (53 años) fue el publicado por Sorahan et al. ${ }^{34}$ en 2004.

\section{Relación causal positiva}

De los catorce estudios seleccionados para esta revisión sistemática, tres ${ }^{24-26}$ de ellos demostraron una relación estadísticamente significativa entre la exposición laboral al cadmio y el desarrollo de neoplasia de próstata (ver tabla III). De éstos, Sharma-Wagner et al. ${ }^{26}$ realizaron el estudio con mayor tamaño muestral encontrado, en el que analizaron una cohorte retrospectiva de trabajadores de diferentes sectores de la industria en Suecia, encontrando un ratio estandarizado de incidencia de neoplasia de próstata elevado en 
trabajadores de la fundición de hierro, plantas de motores y mantenimiento industrial, así como en industrias productoras de jabones y perfumes, en las cuales, entre otros agentes carcinogénicos, se ha observado la exposición al cadmio. Por su parte, Van der Gulden et $a l .^{24}$ realizaron un estudio de casos y controles en trabajadores de la industria y la agricultura en Holanda, y en los siete casos que reportaron una exposición frecuente al cadmio, se halló un OR de 2,76 con un IC al 95\% de 1,05-7,27, asociación que no se observa en aquellos trabajadores que refirieron una exposición ocasional al cadmio. Asimismo, Elghany et al..$^{25}$ realizaron un estudio de casos y controles en trabajadores de materias primas, industria y sector servicios, encontrando una asociación estadísticamente significativa entre neoplasia de próstata y exposición al cadmio (tanto ocupacional como dietética o por hábito tabáquico) con un OR de 1,7 y un IC al 95\% de 1,0-3,1, con diferente proporción según sector.

\section{Relación causal negativa}

Siete $^{27-33}$ de los artículos seleccionados no lograron demostrar que existiera asociación entre la exposición ocupacional al cadmio y el desarrollo de neoplasia de próstata (ver tabla IV).

En el estudio publicado por Armstrong et al. ${ }^{32}$ se halló un riesgo ligeramente elevado de neoplasia de próstata por exposición media o alta al cadmio (OR 1.55 para la exposición media con un IC al 95\% entre 0.49-4.93 y OR 1.35 con un IC al 95\% entre 0.31-5.91 para la exposición alta, no estadísticamente significativo). Fritschi et al. ${ }^{33}$, por su parte, no encontraron asociación estadísticamente significativa de la neoplasia de próstata con ninguna de las exposiciones laborales que analizaron en su estudio.

Otros estudios no hallaron relación entre la neoplasia de próstata y la exposición al cadmio, sin embargo sí observaron una asociación estadísticamente significativa con otras sustancias, como el plomo (Neslund-Dudas et $a l .^{27}$ ), los combustibles diésel (Seidler et $a l . .^{28}$ ), radioisótopos (Rooney et $a l .^{29}$ ), productos de combustión, colorantes, tintes y aceites de corte (Krech et al. ${ }^{30}$ ); o con ocupaciones concretas, tal como observaron Checkoway et al. ${ }^{31}$ en relación con el sector agrario, aunque sin asociación con exposición a pesticidas o herbicidas.

De los cuatro estudios seleccionados que analizaron la mortalidad por diferentes causas en trabajadores (ver tabla V), en aquel publicado por Sorahan et al. en $2004^{34}$ se halló un ratio estandarizado de mortalidad (Standardized Mortality Ratio, SMR) por neoplasia de próstata - así como de pulmón - elevado entre trabajadores expuestos al cadmio, no estadísticamente significativo (muertes: 9 observadas, 7.5 esperadas, SMR: 116). Los tres estudios restantes ${ }^{35-37}$ no lograron demostrar esta relación.

\section{DISCUSIÓN Y CONCLUSIONES}

De acuerdo con el objetivo principal de esta revisión sistemática se realizó un análisis de la literatura científica sobre la neoplasia de próstata asociada a la exposición laboral al cadmio y/o sus compuestos.

Los datos obtenidos en relación con el índice de Burton-Kleber y el índice de Price indicaron una obsolescencia mayor a la esperada en el campo de las ciencias de la salud $^{38,39}$, lo que pone de relieve la necesidad de actualizar los estudios relacionados con la exposición laboral al cadmio y sus compuestos.

Durante la revisión se encontraron estudios observacionales en trabajadores de diferentes sectores de la industria que implicaban una exposición al cadmio. A pesar de no ser el objetivo principal de este trabajo, se decidió incluir en esta revisión los estudios hallados que analizaron la mortalidad por diversas causas ${ }^{34-37}$, puesto que, entre otras, estudiaron la relación entre exposición ocupacional al cadmio y la neoplasia de próstata. De todos los artículos revisados, sólo cinco $^{24-26,32,33,34}$ encontraron relación entre la neoplasia de próstata y la exposición al cadmio y/o sus compuestos, sin embargo esta 
asociación sólo resultó estadísticamente significativa en tres ${ }^{24,25,26}$ de los casos. Van der Gulden et al. ${ }^{24}$ encontraron un aumento de riesgo estadísticamente significativo para el desarrollo de neoplasia de próstata en trabajadores que refirieron una exposición frecuente al cadmio; Sharma-Wagner et al. ${ }^{26}$ hallaron un ratio estandarizado de incidencia de neoplasia de próstata elevado en trabajadores expuestos al cadmio, y Elghany et al. ${ }^{25}$ observaron una asociación estadísticamente significativa entre neoplasia de próstata y exposición ocupacional al cadmio. Además, registraron el tiempo de exposición en las diferentes ocupaciones estudiadas, y cruzaron estos datos con la historia de tabaquismo y exposición al cadmio a través de la dieta, hallando una relación entre exposición alta al cadmio por las diversas vías descritas y la aparición de tipos más agresivos de neoplasias.

Al analizar la historia laboral de los individuos incluidos en la muestra, se los clasificó según sector y se especificó después la profesión. Tanto Van der Gulden et al. ${ }^{24}$ como Elghany et al. ${ }^{25}$ no encontraron un exceso de riesgo en ninguna profesión específica. Por su parte, Sharma-Wagner et al. ${ }^{26}$ hallaron un riesgo aumentado en trabajadores de la industria del jabón, perfume, agricultores y manufactura del tabaco. Sin embargo, en otras profesiones donde existe una exposición a altos niveles de cadmio como la minería, cantería, industria del plástico y la construcción y venta del metal, no se halló un exceso de riesgo específico.

Los resultados de la presente revisión están limitados por las carencias propias de cada trabajo revisado. Según la US Agency for Health Research and Quality ${ }^{40}$, los diseños epidemiológicos de los estudios seleccionados en esta revisión (cohortes y casoscontroles) aportan un nivel de evidencia y grado de recomendación IIb y III respectivamente, de modo que no se puede asegurar por completo la validez y fiabilidad de las observaciones. La recopilación de datos en los estudios de casos y controles se realizó a través de cuestionarios que se entregaron a los individuos incluidos en la muestra o entrevistas, incurriendo así en sesgos, lo que pudo llevar a subestimar una posible asociación existente. Además la mayoría de los estudios no describieron mediciones cuantitativas (salvo Sorahan et al..$^{37}$, que reportaron una concentración estimada de cadmio ambiental en ciertos sectores de la fábrica que estudiaron en 1949 de 0.6 to $2.8 \mathrm{mg} / \mathrm{m}^{3}$. Refirieron además que tras la instalación de un sistema de ventilación en 1950 estas concentraciones consiguieron reducirse a $0.5 \mathrm{mg} / \mathrm{m}^{3}$ en la práctica totalidad de la fábrica). Por otro lado en la mayoría de los estudios tampoco se especificó la duración de la exposición al cadmio y/o sus compuestos (limitándose en la mayoría de los casos a clasificarla en "alta, media y baja"32, "expuesto o no expuesto"30,31 o "frecuente, ocasional e infrecuente"24) y en algunos de ellos no se realizó ningún control de los factores de confusión (ingesta dietética y hábito tabáquico) que pudieran afectar a sus resultados ${ }^{32}$. Otro punto a señalar es la dificultad para atribuir la neoplasia de próstata específicamente a la exposición al cadmio, ya que en algunos ambientes laborales el contacto con este contaminante ocurre de forma simultánea al de otros compuestos como el plomo, el cromo o el zinc, que también tienen un efecto carcinogénico sobre la próstata ${ }^{41}$.

A pesar de haber encontrado un reducido número de estudios originales que corroboren la existencia de una relación estadísticamente significativa entre la exposición ocupacional al cadmio y el desarrollo de neoplasia de próstata en esta revisión sistemática, existen otros trabajos que sí han encontrado esta relación, como el metaanálisis publicado por Ju-Kun et al. ${ }^{12}$ en 2016 , donde se halló una asociación positiva entre la alta exposición al cadmio y el riesgo de neoplasia de próstata para la exposición ocupacional, pero no para la exposición no ocupacional.

Por todo lo anteriormente expuesto, y a pesar de encontrar resultados reportando enfermedad tras exposición al cadmio y/o sus compuestos, no se puede concluir que exista una relación directa entre la aparición de neoplasia de próstata y la exposición laboral al cadmio y/o sus compuestos, por lo que serían necesarios nuevos estudios, con mayor tamaño muestral, que cuantifiquen el tiempo de exposición e incluyan mediciones cuantitativas de las concentraciones de cadmio, y que de esta manera aporten mayor evidencia acerca de los posibles efectos carcinogénicos del cadmio y/o sus compuestos en el ambiente laboral. 


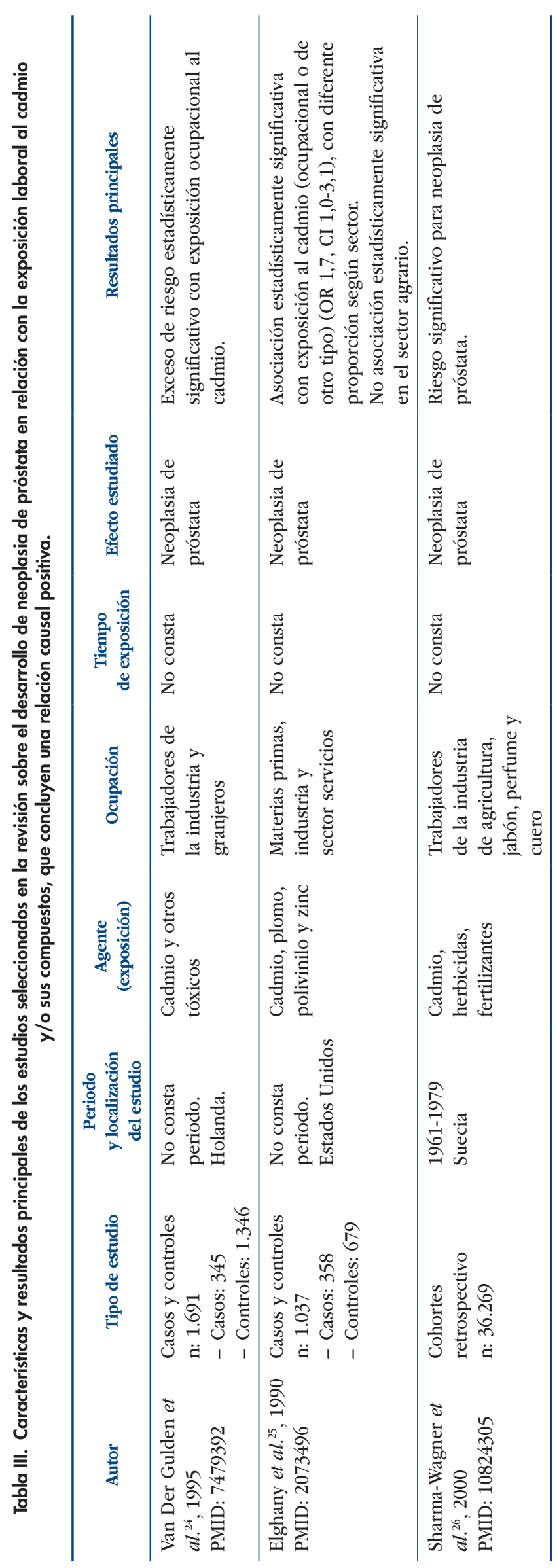




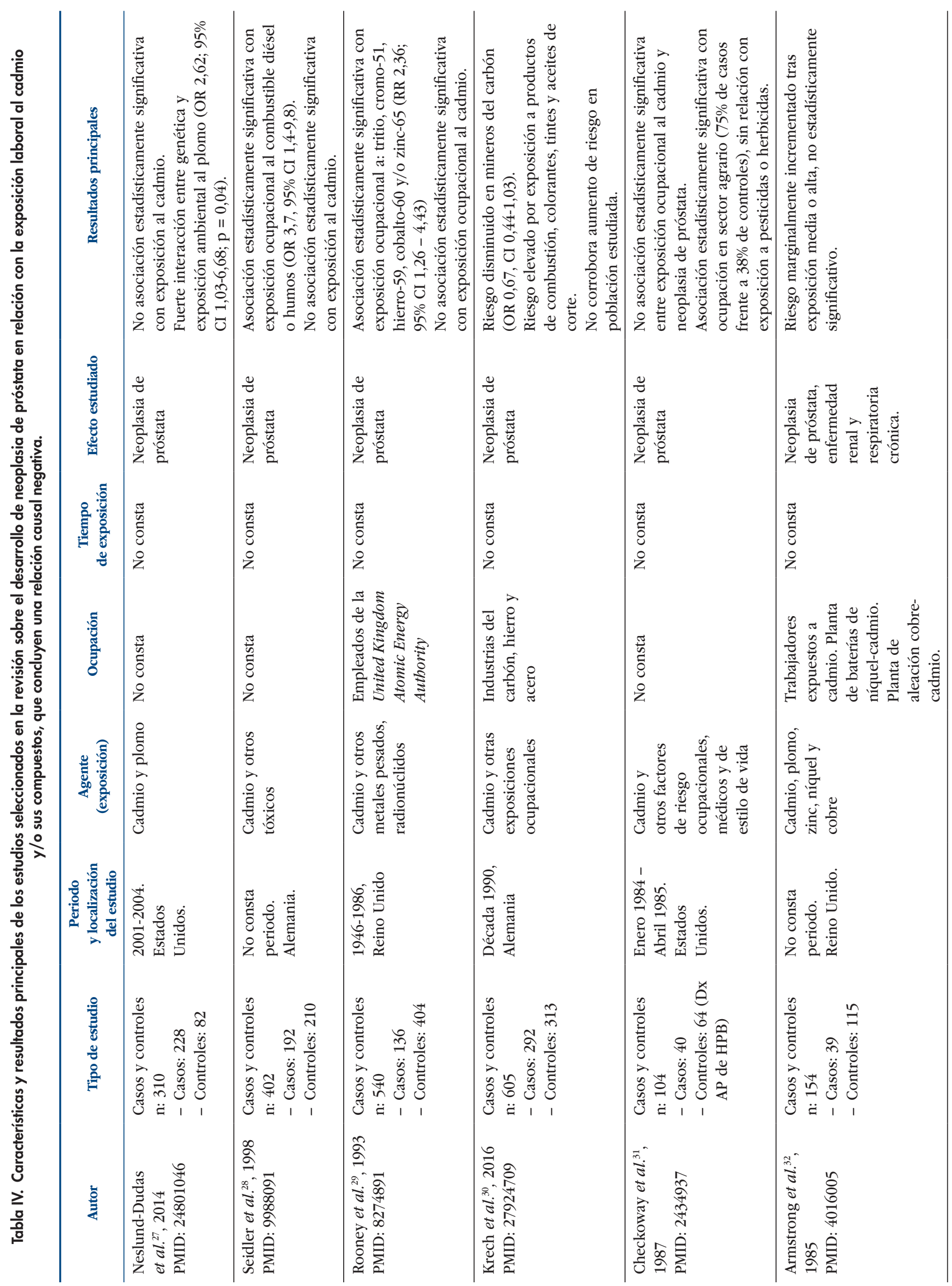



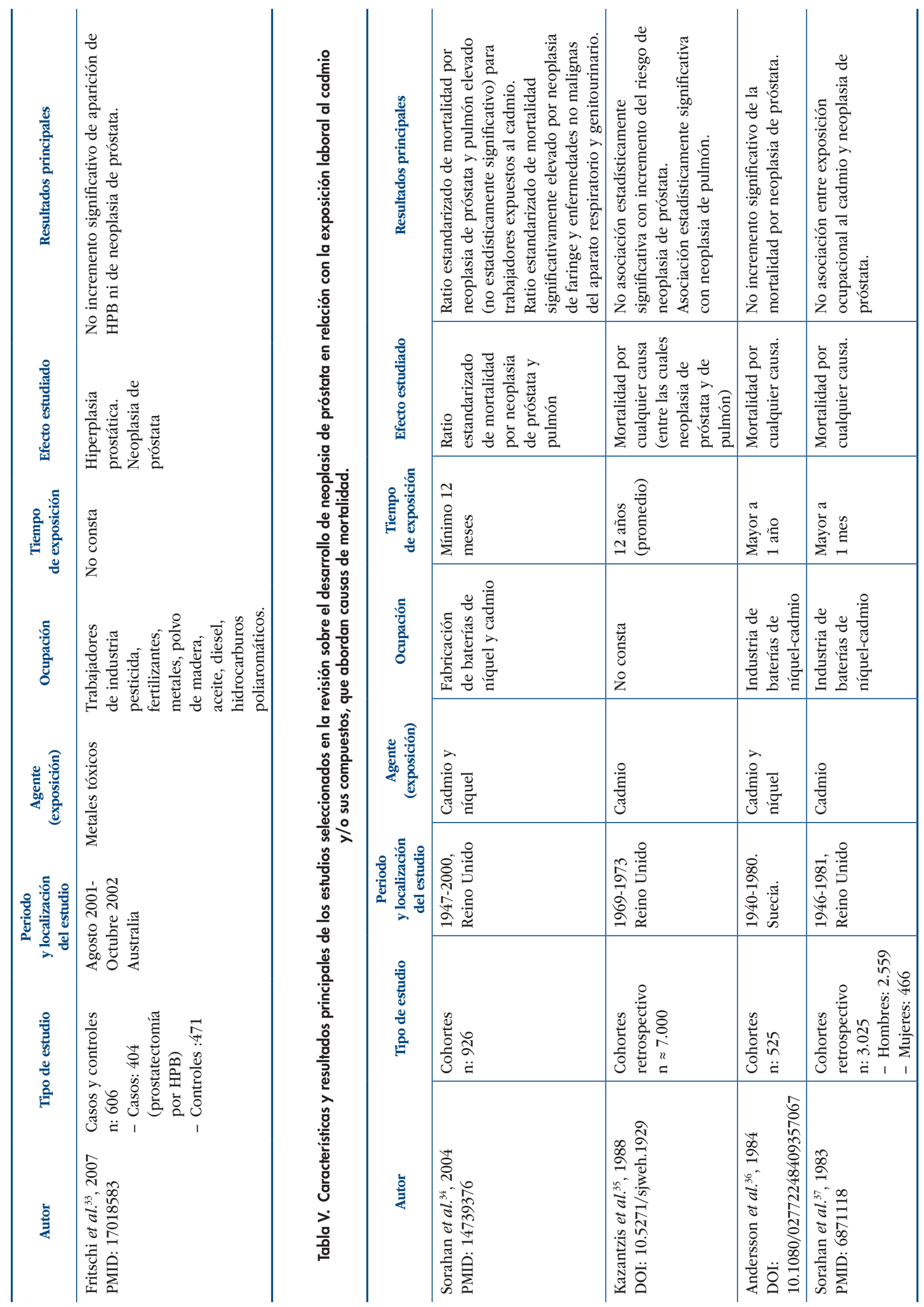


\section{REVISIÓN BIBLIOGRÁFICA}

1. Jemal A, Bray F, Center M. Global Cancer Statistics. Ca Cancer J Clin. 2011;61(2):69-90. DOI: 10.3322/ caac. 20107

2. Bashir MN. Epidemiology of Prostate Cancer. Asian Pac J Cancer Prev. 2015;16(13):5137-41. DOI: 10.7314/ APJCP.2015.16.13.5137

3. Siegel RL, Miller KD, Jemal A. Cancer statistics. Ca Cancer J Clin. 2015 Jan;65(1):5-29. DOI: 10.3322/ caac. 21254

4. Galceran J, Ameijide A, Carulla M. Cancer incidence in Spain, 2015. Clin Transl Oncol. 2017;19(7):799-825. DOI 10.1007/s12094-016-1607-9.

5. Allott EH, Masko EM, Freedland SJ. Obesity and prostate cancer: weighing the evidence. Eur Urol. 2012;63(5):800-9. DOI: 10.1016/j.eururo.2012.11.013.

6. Venkateswaran V, Klotz LH. Diet and prostate cancer: mechanisms of action and implications for chemoprevention. Nat Rev Urol. 2010;7(8):442-53. DOI: 10.1038/nrurol.2010.102.

7. IARC Working Group on the Evaluation of Carcinogenic Risk to Humans [monograph on the Internet] Beryllium, Cadmium, Mercury, and Exposures in the Glass Manufacturing Industry. Lyon, France: International Agency for Research on Cancer; 1993. (IARC Monographs on the Evaluation of Carcinogenic Risks to Humans, No. 58.) [cited 2019 Jan 31]. Available from: https://bit.ly/2SCVKWw

8. Amzal B, Julin B, Vahter M, Wolk A, Johanson G, Åkesson A. Population Toxicokinetic Modeling of Cadmium for Health Risk Assessment. Environ Health Perspect. 2009;117(8):1293-301. DOI: 10.1289/ ehp.0800317.

9. Järup L, Åkesson A. Current status of cadmium as an environmental health problem. Toxicol Appl Pharmacol. 2009;238(3):201-8. DOI: 10.1016/j.taap.2009.04.020.

10. National Toxicology Program: 14th Report on Carcinogens [Homepage]. National Toxicology Program (NTP); 2016. [cited 2019 Jan 16]. Available from: https://bit.ly/2EJ3ZMR

11. Directiva 2002/95/CE del Parlamento Europeo y del Consejo sobre restricciones a la utilización de determinadas sustancias peligrosas en aparatos eléctricos y electrónicos. Diario oficial de la UE, $\mathrm{n}^{\circ}$ L37/19, (13.02.2003) [cited 2019 Jan 16]. Available from: https://bit.ly/2UibSyf

12. Ju-Kun S, Yuan D-B, Rao H-F. Association Between Cd Exposure and Risk of Prostate Cancer: A PRISMACompliant Systematic Review and Meta-Analysis. Medicine (Baltimore). 2016;95(6):e2708. DOI: 10.1097/ MD.0000000000002708.

13. Cheung MR, Kang J, Ouyang D, Yeung V. Association between Urinary Cadmium and All Cause, All Cancer and Prostate Cancer Specific Mortalities for Men: an Analysis of National Health and Nutrition Examination Survey (NHANES III) Data. Asian Pac J Cancer Prev. 2014;15(1):483-8. DOI: 10.7314/ APJCP.2014.15.1.483

14. Julin B, Wolk A, Johansson J-E. Dietary cadmium exposure and prostate cancer incidence: a populationbased prospective cohort study. Br J Cancer. 2012;107(5):895-900. DOI: 10.1038/bjc.2012.311.

15. Kjellstrom T, Friberg L, Rahnstert B. Mortality and Cancer Morbidity among Cadmium-Exposed Workers. Environ Health Perspect. 1979;28:199-204. DOI: 10.1289/ehp.28-1637490

16. Vinceti M, Venturelli M, Sighinolfi C. Case-control study of toenail cadmium and prostate cancer risk in Italy. Sci Total Environ. 2007;373(1):77-81. DOI: 10.1016/j.scitotenv.2006.11.005.

17. West DW, Slattery ML, Robinson LM. Adult Dietary Intake and Prostate Cancer Risk in Utah: A CaseControl Study with Special Emphasis on Aggressive Tumors. Cancer Causes Control. 1991;2(2):85-94.

18. Chen Y-C, Pu YS, Wu H-C. Cadmium burden and the risk and phenotype of prostate cancer. BMC Cancer. 2009;9:429. DOI: 10.1186/1471-2407-9-429.

19. García-Esquinas E, Pollan M, Tellez-Plaza M. Cadmium Exposure and Cancer Mortality in a Prospective Cohort: The Strong Heart Study. Environ Health Perspect. 2014;122(4):363-70. DOI: 10.1289/ehp.1306587

20. Sawada N, Iwasaki M, Inoue M. Long-term Dietary Cadmium Intake and Cancer Incidence. Epidemiology. 2012;23(3):368-76. DOI: 10.1097/EDE.0b013e31824d063c.

21. IARC Working Groups [monograph on the Internet]. IARC Monographs on the Identification of Carcinogenic Hazards to Humans, Volume 100 C, Cadmium and Cadmium Compounds; 2012. [cited 2019 Jan 16]. Available from: https://bit.ly/2VmSWOY

22. Wanden-Berghe C, Sanz-Valero J. Systematic reviews in nutrition: standardized methodology. Br J Nutr. 2012;107(Suppl 2):S3-7. DOI: 10.1017/S0007114512001432. 
23. von Elm E, Altman DG, Egger M, Pocock SJ, Gøtzsche PC, Vandenbroucke JP. The Strengthening the Reporting of Observational Studies in Epidemiology (STROBE) Statement: Guidelines for reporting observational studies. Int J Surg. 2014;12(12):1495-9. DOI: 10.1136/bmj.39335.541782.AD

24. van der Gulden JW, Kolk JJ, Verbeek AL. Work environment and prostate cancer risk. Prostate. $1995 ; 27(5): 250-257$.

25. Elghany NA, Schumacher MC, Slattery ML. Occupation, cadmium exposure, and prostate cancer. Epidemiology. 1990;1(2):107-15.

26. Sharma-Wagner S, Chokkalingam AP, Malker HS. Occupation and prostate cancer risk in Sweden. J Occup Environ Med. 2000;42(5):517-25.

27. Neslund-Dudas C, Levin AM, Beebe-Dimmer JL. Gene-environment interactions between JAZF1 and occupational and household lead exposure in prostate cancer among African American men. Cancer Causes Control. 2014;25(7):869-79. DOI: 10.1007/s10552-014-0387-1

28. Seidler A, Heiskel H, Bickeböller R. Association between diesel exposure at work and prostate cancer. Scand J Work Environ Health. 1998;24(6):486-94. DOI: 10.5271/sjweh.373

29. Rooney C, Beral V, Maconochie N. Case-control study of prostatic cancer in employees of the United Kingdom Atomic Energy Authority. BMJ. 1993;307(6916):1391-7.

30. Krech S, Selinski S, Bürger H. Occupational risk factors for prostate cancer in an area of former coal, iron, and steel industries in Germany - Part 2: results from a study performed in the 1990s. J Toxicol Environ Health A. 2016;79(22-23):1130-5. DOI: 10.1080/15287394.2016.1219603.

31. Checkoway H, Diferdinando G, Hulka BS. Medical, life-style, and occupational risk factors for prostate cancer. The Prostate. 1987;10(1):79-88.

32. Armstrong BG, Kazantzis G. Prostatic cancer and chronic respiratory and renal disease in British cadmium workers: a case control study. Occup Environ Med. 1985;42(8):540-5.

33. Fritschi L, Glass DC, Tabrizi JS. Occupational risk factors for prostate cancer and benign prostatic hyperplasia: a case-control study in Western Australia. Occup Environ Med. 2006;64(1):60-5. DOI: 10.1136/oem.2006.027706

34. Sorahan T, Esmen NA. Lung cancer mortality in UK nickel-cadmium battery workers, 1947-2000. Occup Environ Med. 2004;61(2):108-16. DOI: 10.1136/oem.2003.009282.

35. Kazantzis G, Lam TH, Sullivan KR. Mortality of cadmium-exposed workers. A five-year update. Scand J Work Environ Health. 1988;14(4):220-3. DOI: 10.5271/sjweh.1929.

36. Andersson K, Elinder CG, Hogstedt C. Mortality among cadmium and nickel-exposed workers in a Swedish battery factory. Toxicol Environ Chem. 1984;9(1):53-62.

37. Sorahan T, Waterhouse JA. Mortality study of nickel-cadmium battery workers by the method of regression models in life tables. Occup Environ Med. 1983;40(3):293-300.

38. Arias FG. Obsolescencia de las referencias citadas: un mito académico persistente en la investigación universitaria venezolana. E-Cienc Inf. 2016;7(1):1. DOI: 10.15517/eci.v7i1.26075

39. Sanz-Valero J, Wanden-Berghe C. Análisis bibliométrico de la producción científica, indizada en MEDLINE, sobre los servicios de salud proporcionados por las unidades de hospitalización a domicilio. Hosp Domic. 2017;1(1):21. DOI: 10.22585/hospdomic.v1i1.3.

40. Rockville (MD): Agency for Healthcare Research and Quality (US) [monograph on the Internet]. Making Health Care Safer II: An Updated Critical Analysis of the Evidence for Patient Safety Practices. (Evidence Reports/Technology Assessments, No. 211.); 2013 [cited 2019 Jan 31]. Available from: https://bit.ly/2TjUCfS

41. International Agency for Research on Cancer, IARC Working Group on the Evaluation of Carcinogenic Risks to Humans, editors [monograph on the Internet]. Inorganic and organic lead compunds. Lyon, France: International Agency for Research on Cancer; 2006. (IARC monographs on the evaluation of carcinogenic risks to humans) [cited 2019 Jan 31]. Available from: https://bit.ly/2tC7xKK 